\title{
El papel de los museos en la sociedad actual: discurso institucional o museo participativo
}

\author{
The Role of Museums in Contemporary Society: \\ Institutional Discourse or Participatory Museum
}

\author{
Isabel Ma GARCíA Fernández \\ Departamento de Pintura y Restauración \\ Facultad de Bellas Artes, Universidad Complutense de Madrid \\ museoig@ucm.es
}

Recibido: 1-04-2014

Aceptado: 10-09-2014

\begin{abstract}
RESUMEN
Desde hace ya varias décadas se ha extendido un lema convertido en habitual año tras año: que los museos necesitan cambios y que, éstos, deben seguir produciéndose en el futuro para que estas instituciones sigan siendo significativas en la sociedad, es decir, para los ciudadanos a quienes representan y sirven. La cuestión de cómo afrontan los cambios es lo que verdaderamente ha dado lugar a reflexiones interesantes en el campo de la museología. No existe un enfoque único para una cuestión tan compleja, ya que está compuesta de múltiples capas que, en muchas ocasiones, son dificilmente acoplables. Sin embargo, en términos generales, podemos apuntar que va a ser en la exposición donde los nuevos planteamientos tendrán su desarrollo.
\end{abstract}

Palabras Clave: Museo participativo. Museo inclusivo. Discurso institucional.

\begin{abstract}
Since a few decades a recurring catchphrase has been accepted: the museums need changes and those will be produced in the future, in order to be socially significant for the citizens they represent and serve. How to face the changes has lead to interesting considerations in the museology field. There is not a unique approach to a complex question, since is composed by multiple layers that often are adjusted with difficulty. However, in general terms, we can point out that the new proposals will arise and develop in the exhibition practice.
\end{abstract}

KEY WORDS: Participatory museum. Inclusive Museum. Institutional Discourse. 


\section{Reflexionando sobre el museo}

En los últimos años en muchas publicaciones de museología los conceptos de museo inclusivo y museo participativo han estado presentes. Recientemente el Presidente del ICOM, el Prof. Dr. Hans-Martin Hinz hacía mención a ello al presentar el lema del Día Internacional de los $\mathrm{Mu}-$ seos 2014, exponiendo que los museos se están enfrentando continuamente a cambios que les hacen reconsiderar su misión tradicional y buscar nuevas estrategias para conectar con sus comunidades, haciendo que las colecciones sean más accesibles para sus usuarios. Así, muchos museos renuevan las formas de presentar sus colecciones para conseguir implicar a la comunidad y mantener el contacto con sus visitantes a través de la memoria compartida, propiciando a su vez, la conexión entre los diferentes tipos de colecciones y sus audiencias ${ }^{1}$.

Estas estrategias pasan por el análisis de la institución, el contexto y la relación entre ambos; en esta línea también en 2014, en la convocatoria del $36^{\circ}$ Simposio Internacional del ICOFOM Nuevas tendencias en Museología, que se celebrará en París, se hace hincapié en la existencia de numerosos estudios de prospectiva museal que cuestionan el futuro de la institución. Estos estudios apuntan a comprender mejor la evolución de esta institución particular ante los cambios significativos de la sociedad actual: aquellos relacionados con las poblaciones, con la tecnología, con los métodos educativos, con las políticas económicas, etc. ${ }^{2}$

En la actualidad, existen publicaciones que proporcionan un enfoque general de la realidad museológica, como el informe elaborado por la Asociación de Museos del Reino Unido en 2013 Museum Change Lives. The MA'S Vision for the impact of Museums. Este documento reconoce el aumento del impacto ${ }^{3}$ que los museos tienen en la sociedad. Este impacto es positivo, proporcionando cambios en la vida de las personas. El punto de partida es la evidencia de esas transformaciones, es decir, la forma en que enriquecen a los individuos, contribuyendo a crear comunidades fuertes y dinámicas y ayudando a generar una sociedad más justa. Los museos, por otra parte, mejoran y se regeneran en gran medida gracias a la capacidad y creatividad de su público ${ }^{4}$.

Estas instituciones que se construyeron para el estudio y conservación de las colecciones, ahora tratan de encontrar el modo de hacerlas más accesibles al público, tanto conceptual como físicamente. Se plantea que estas instituciones deben ser "ambiciosas" a la hora de considerar su contribución en la sociedad. A pesar de las difíciles circunstancias que nos han tocado vivir, hoy en día, se pide a los museos que reflexionen sobre su misión y objetivos y que se conviertan de verdad en instrumentos que mejoren la vida de los ciudadanos, que no solo proporcionen un refugio placentero que nos evada de la vida cotidiana, ni sean simples repositorios de conocimientos y bienes patrimoniales ${ }^{5}$. Debe existir un compromiso que contemple y trate de armonizar los intereses y necesidades de los individuos y la sociedad con el contexto en el que se desarrolla; es decir, ponerse a su servicio (sirva como ejemplo apoyar a los artistas), implicarse en la preservación de las tradiciones y servir de apoyo a la educación de todo tipo de público. En definitiva, las instituciones museísticas se deben preguntar cómo pueden convertirse en relevantes para su público y comunidad y determinar cuál puede ser su aportación.

Por otro lado, desde hace tiempo los museos buscan el debate y el cuestionamiento a través de la reflexión crítica y pretenden implicarse en cuestiones contemporáneas (ejemplos de tipo social y ético son la discriminación, la pobreza, la exclusión...); asimismo, intentan estimular la contemplación, la curiosidad y la creatividad. Ya se apuntaba en la década de los 70 del siglo pasado que los museos son más foros que templos $^{6}$. Cameron (1972:198) afirmaba que el foro es donde tienen lugar las batallas y el templo es donde descansan las victorias, pensaba que esos dos aspectos no podían fundirse, ya que si se llevaba el foro -entendiendo al foro como un lugar de confrontación, innovación y experimentación- dentro del templo su efecto se inhibiría y se castraría y, si lo hacían, tendrían consecuencias explosivas. Por otro lado, también pensaba que los museos, independientemente de su tipología, poseen el conocimiento y recursos para interpretar cuestiones que son de importancia pública, no importa que sean controvertidas, ya que es su deber y no pueden quedarse al margen (Cameron 1972: 199). Recientemente, Nicholas Serota, director de los museos y galerías Tate, volvía a referirse a los museos como foros, pero también como cajas de tesoros ${ }^{7}$. Una descripción que aúna la tradición y el origen de estas instituciones y su papel en la sociedad contemporánea. Definición extraña y hasta podríamos decir que algo desfasada, si tenemos en cuenta que Stephen E. Weil (1995:8), hace casi vein- 
te años ya, planteaba que si llamas a un museo casa de tesoros su objetivo será elegir cuidadosamente objetos de gran rareza y valor. Decía, llámalo laboratorio para las artes visuales y lo que se puede esperar es ver lo inusual, lo nuevo, lo experimental. Comparado con el museo templo, el museo laboratorio es un lugar donde la verdad y la belleza se descubren; no mediante la revelación, sino a través de la prueba y el error pacientes... La institución que nosotros imaginamos es completamente diferente, posee una vertiente intelectual que los templos o las casas museo no tienen ${ }^{8}$. Hoy se apuesta por la imagen de foro que incorpora también la de laboratorio, mucho más acorde con la idea contemporánea de la cultura participativa.

Que los museos siguen siendo instituciones muy valoradas es una realidad. Aun con las precisiones que habría que hacer, el éxito de los museos parece sorprendente si tenemos en cuenta que hoy el público tiene más opciones de ocio que nunca. Parte de este entusiasmo se entiende por los cambios que ya se han producido en los visitantes y lo que esperan de estas instituciones. En un artículo del Economist, de diciembre de $2013^{9}$, se exponía el dato de la existencia de casi 55.000 museos en el mundo, más del doble que hace 20 años; muchos se abren en China, donde se inauguraron más de 450 en un año. La mayoría de los nuevos museos es de arte contemporáneo. También se ha registrado un récord de visitantes en algunos países y en instituciones concretas; hay que destacar al museo del Louvre que alcanzó los 10 millones de visitas en 2012. En España, el Museo con más afluencia en 2013 fue el Museo Nacional Centro de Arte Reina Sofía. Hay que apuntar, no obstante, que en el caso del Louvre es una tendencia consolidada, y, en el caso del museo Reina Sofía es coyuntural, ya que se produce por el interés despertado por la exposición temporal dedicada a Dalí ${ }^{10}$. No obstante, como dice su director, hay que ser positivos "en la tendencia general se sigue observando un gran interés por parte del público hacia las ofertas culturales de los museos y apuesta por "trabajar con las múltiples minorías"11. Ahondando en esta cuestión, desde el mundo profesional se ha planteado la inclusión y la participación del público como dos aspectos fundamentales en el panorama contemporáneo. Hernández (2011:410) exponía que

"los museos están llamados a valorar y favorecer la diversidad cultural como un elemento esencial para la sociedad en la que se han de tener en cuenta valores tan importantes como el diálogo y la aceptación de los demás basados en el pluralismo, la diferencia, la competencia y la creatividad. Solamente desde ellos pueden darse el encuentro, los intercambios y la reflexión comunitaria".

Una de las iniciativas es la que plantea la $\mathrm{Mu}$ seum Association del Reino Unido (2013): enganchar y entusiasmar al público que va a los museos. Ésta se ha convertido en una máxima en el campo de la museología; sin embargo, nos preguntamos si de verdad el museo es accesible a todos y ofrece un contexto en el que los visitantes se sienten identificados, y si, desde los museos, se puede ayudar a la gente a desarrollar sus habilidades, confianza y autoestima, además de integrar a colectivos para que no se sientan marginados. Cabría preguntarse entonces si las instituciones están realizando un ejercicio de autorreflexión y tienen claras cuáles son las ofertas culturales más apropiadas, qué es realmente el valor público y qué aportan. La realidad es que muchos visitantes demandan "experiencias significativas" y exigen que se aborden cuestiones contemporáneas; es decir, esperan conectar intelectualmente y emocionalmente con los contenidos y actividades que se ofrecen participando activamente.

La nueva mirada al papel de los museos y cómo encaran el futuro se fundamenta en buena medida en los denominados "estudios de impacto" o de efectos producidos en la opinión pública por los distintos acontecimientos. Algunos de estos estudios se basan en las preferencias de ciertos grupos de edad, como los Millennials en Estados Unidos que son los nacidos entre 1979 y $1994^{12}$ y que, según un estudio, no están interesados en las instituciones y organizaciones, sino más en la gente y en las ideas concretas que les parecen importantes, y que ellos eligen apoyar y ayudar. Las donaciones a instituciones sin ánimo de lucro están siendo cuestionadas por los donantes, particularmente por los más jóvenes que exigen resultados medibles a cambio de su dinero. Existe, por tanto, un incremento de la tendencia de los donantes a centrarse en las contrapartidas monetarias, más que a tener en cuenta las necesidades sociales. Cómo esto afecta al museo es difícil de especificar; por un lado, tiene una implicación económica, ya que los museos se están quedando fuera de sus donaciones y, por otro, afecta a la misión y objetivos de las instituciones que tienen que revisarlos para estar en sintonía con las nuevas demandas. 


\section{Discurso institucional}

No vamos a preguntarnos a estas alturas qué es un museo, pero sí es importante saber qué ofrece. Hace tiempo que se acepta que la importancia del museo depende del valor de sus objetivos y su capacidad para conectar con el público, no del mero hecho de ser un museo o de su potencial tecnológico.

Los museos son lugares importantes en los que la sociedad puede identificarse y presentarse públicamente. Los museos, en sus correspondientes declaraciones de objetivos, exponen sus valores educativos y su papel y responsabilidad respecto al público y sus colecciones. Todo ello evoluciona y cambia y tiene que ser revisado. Como hemos apuntado más arriba, algunos estudios han evidenciado que en estas instituciones se producen descubrimientos, se comparten conocimientos, se inspiran ideas y nuevas visiones, incentivando la curiosidad de los visitantes que descubren más sobre aspectos que tienen que ver con la historia, el arte, la ciencia y la vida contemporánea. La mayoría del público que va a los museos los considera lugares donde se dan ideas estimulantes y donde tiene lugar un aprendizaje activo ${ }^{13}$.

También se puede afirmar que los museos consolidan cultura, la dotan de tangibilidad, algo que otras instituciones no pueden hacer. Las definiciones, por regla general, enfatizan el servicio público de estas instituciones (Dubin 1999:3) ${ }^{14}$ ya que de ahí radica su importancia e interés.

Uno de los grandes debates se centra en la autoridad del museo, que tiene que ver con la reputación; es decir, con su relevancia en la sociedad. Esta relevancia viene de su relación con la comunidad a quien sirve. La gente confía en los museos y espera que les proporcionen información veraz. El cuestionamiento y el diálogo que lleva a la generación de conocimiento ha sido un objetivo claro de estas instituciones. No obstante, la proliferación de información sobre ellas en distintos canales hace que ni siquiera sean los propios museos las fuentes de información sobre sus contenidos. Stephen E. Weil (1995: xiii) planteaba que no importaba cuánto la comunidad museológica pueda insistir en su propia objetividad, la realidad es que el sentido de cada museo se conforma en último término por la autoridad dominante bajo la que opera. Los museos, por tanto, no son neutrales -no pueden, ni deben serlo-, se acepta que las políticas del pasado y del presente han dado forma a las colecciones, información e interpretación ${ }^{15}$.
Se ha apuntado en muchas ocasiones que los museos son espacios politizados donde pueden tener lugar todo tipo de "dramas", entendiéndose que pueden ser objeto de una gran variedad de conflictos de intereses y restricciones. Incluso se ha llegado a decir que todo lo que hace un museo es político (aunque no sea de una manera consciente), esforzándose en producir productos y significados culturales en consonancia con lo que se espera de ellos. Los museos son vulnerables, son observados y cuestionados, aunque muchas veces sin criterio. La dependencia de las críticas merma sus defensas, al existir un gran número de puntos de presión y conexiones con múltiples agentes (sociales, políticos, económicos, etc.) se crean limitaciones que restringen la supuesta libertad de las instituciones. Estas críticas en muchas ocasiones vienen de dentro de la institución cuestionando la visión única, la voz unánime sobre las cuestiones que se abordan que no tienen en cuenta otras posibles perspectivas.

Hooper-Greenhill según recoge Tony Bennet (2006:103) planteaba la existencia de una división entre el espacio oculto del museo, donde se produce y organiza el conocimiento, y los espacios públicos, en los que se ofrece para consumo pasivo, provocando un discurso monológico dominado por la voz autoritaria del museo. Hace tiempo que se aboga porque los museos se conviertan en los catalizadores de nuevas ideas y conversaciones y sirvan como moderadores, más que como simples presentadores de contenidos. Los grupos minoritarios deben estar representados y tener la oportunidad de compartir sus experiencias. Ahora se busca incluir más voces y vivencias para acceder a una realidad más completa.

\section{El museo participativo}

A finales de los años 60 y durante la década de 1970 se puede decir que los museos empezaron a salir de su cascarón y comenzaron a mirar a su alrededor e intentar involucrar a la gente en la comunidad (museología comunitaria). Cuarenta años más tarde estamos hablando de que el museo tiene la oportunidad de reinventarse como institución y cambiar la relación con su público inmerso en la llamada cultura participativa. La cultura participativa, según Henry Jenkins (2009: xi), es una cultura con relativas pocas barreras para la expresión artística y el compromiso ciudadano y cuenta con un apoyo fuerte para crear y compartir las creaciones de los individuos; asimismo, requiere 
algún tipo de orientación informal donde los más experimentados ayudan a los principiantes. En una cultura participativa, sus miembros también creen en la validez de sus contribuciones y sienten algún tipo de conexión social con los otros (al menos sus miembros se preocupan de las opiniones de otros sobre los que han creado). También plantea Jenkins (2009: 8) que la cultura participativa está emergiendo como la cultura que absorbe y responde a la explosión de las nuevas tecnologías en los medios que hacen posible que el consumidor medio pueda archivar, anotar, apropiar y recircular los contenidos de esos medios de manera influyente ${ }^{16}$.

Mientras que la cultura participativa ha tenido un desarrollo de más de 20 años y parece que ya ha calado en la sociedad, también el museo participativo empieza a hacerlo, por lo menos, entre los profesionales ${ }^{17}$. ¿Cómo pueden los museos reconectar con el público y demostrar su valía y relevancia en la vida contemporánea? Una manera, como plantea Nina Simon en su libro The Participatory Museum, es invitar a la gente a involucrarse activamente como participantes culturales y no consumidores pasivos ${ }^{18}$. Precisamente el museo participativo parte de la existencia de un gran vacío entre los intereses del público, sus preferencias y lo que ofrece el museo, y aquí radica su reto. Hay todavía muchas personas que no visitan museos, las razones son variadas, pero un número importante se siente excluido, piensa que son espacios culturales que no están hechos para ellos. Por otro lado, las plataformas como la Web 2.0 han fomentado que una parte del público que sí visita los museos no solo desee participar, sino que espera tener más oportunidades para conectar, contribuir, comunicar y crear en el contexto museístico. No obstante, volvemos a señalar que hay todavía mucho trabajo que hacer para que estas instituciones consigan atraer a la gente y consigan su participación.

En la actualidad, se considera que el visitante tiene la misma importancia que la colección y, por ello, su experiencia en la institución es esencial. En este sentido, Samis y Michaelson (2013: 54) se preguntan cuáles son los mecanismos para conseguir los objetivos de un museo, una vez que éste decide cambiar su enfoque y situar a los visitantes en el centro de su misión. Hacemos referencia entonces al término Audience engagement nacido de la necesidad de conectar al público con el museo y dar cabida a sus intereses antes y después de su visita, teniendo en cuenta que no buscan solo "ir" a eventos culturales. ${ }^{19}$
Se puede decir que en los planteamientos tradicionales, los museos fundamentalmente proporcionan contenidos para que los consuma el público sin ningún tipo de cuestionamiento. En los proyectos participativos, como afirma Simon (2010), la institución apoya experiencias multidireccionales sobre su contenido; además sirve de plataforma que conecta diferentes usuarios que actúan como creadores, distribuidores, consumidores, críticos y colaboradores. Para ello, se debe adoptar un nuevo lenguaje y aplicar nuevas ideas y perspectivas, teniendo en cuenta que la participación no solo debe ser externa, sino también interna.

\section{Discurso institucional, museo participativo y la exposición}

En el proceso de mostrar o exponer es donde se establecen las relaciones entre el museo y el visitante. Las exposiciones son parte importante del museo, puede haber exposiciones sin museo, pero no puede haber museos sin exposiciones.

\subsection{Temas}

En términos generales, hasta hace poco se podía afirmar que los museos raramente abordaban cuestiones políticas, o al menos, pretendían no abordarlas. Hoy sí se plantea afrontar las verdades controvertidas o complejas, conectar los objetos pasados con cuestiones del presente e incluso mostrar hechos incómodos y truculentos. Se ha dicho repetidamente que los museos poseen las mejores historias de la humanidad, cómo las contamos es la gran cuestión.

Los museos han presentado historias sobre el poder, y las respuestas han sido también muestras de poder: ofensivas, defensivas o asertivas que reclamaban cambios. En los años 90 se postuló como tema importante el revisionismo histórico, tanto a nivel del individuo, como de la sociedad. La nueva historia social ya no se escribe desde la perspectiva del rico y el poderoso, sino desde la perspectiva de la gente corriente que se convierte en tema, constituyendo un reto para el museo -aun así, no todo el mundo se siente identificado-.

La gran cuestión es que las historias necesariamente sacrifican la verisimilitud de los hechos en interés de dar una forma definida controlada que tenga un sentido hasta el final. En ocasiones, las historias que se cuentan en el museo pueden distorsionar la realidad; ésta es demasiado rica y 
densa para presentarse toda. La solución no es dejar de contar historias, sino reconocer lo que son: una versión, pero de ninguna manera la única versión o necesariamente la versión verdadera. En definitiva, debemos comprender más sobre las historias que contamos, las que dejamos fuera y las que se modifican.

\subsection{Discurso}

Hoy en día, se plantea cómo cambiar los hábitos del público que, después de un entrenamiento cultural sólido visitando exposiciones monológicas, ya no cree en sus valores, habilidades o incluso en la propiedad de sus propias iniciativas. Mieke Bal (2008: 537) propone como reto educar al público no formado estéticamente y adicto a la lectura de las cartelas en la pared.

Las exposiciones han pasado de estar enfocadas a los objetos para centrarse en las ideas, la mayoría de las veces existe un componente educativo, de compromiso con cuestiones de interés. La gente actualmente quiere contar sus historias y no que otros interpreten sus experiencias. Hay un debate sobre quién está autorizado a hablar de quien o de qué, surgiendo el debate de la representación. La pregunta actual es inecesitamos una voz institucional? Desde la cultura participativa se aboga por compartir el contenido diverso, personalizado y cambiante coproducido con el público en lugar de proporcionar el mismo contenido a todo el mundo, cambiando así las historias "sobre" algo a "para" alguien.

La frontera entre el conocimiento que se produce dentro del museo y en otras partes se está borrando; algunos apuntan a que los museos actuarán como "facilitadores", abriéndose a interpretaciones con contenidos generados por los usuarios; ello incrementará el sentido de pertenencia y aumentará la relación del público con los museos.

\subsection{Tecnología}

Los contenidos de los museos se pueden hacer accesibles a través del uso de la tecnología y reutilizarse en la creación de conocimientos. Cómo gestionamos y diseminamos la información digital debe favorecer la inclusión. El sistema del museo participativo, según Simon (2010), plantea la interacción a diferentes niveles entre los visitantes con intereses comunes.

Ya existen varias iniciativas en el mundo de los museos sobre todo de arte ${ }^{20}$ donde se experimen- ta con la tecnología para llegar al público más allá del espacio físico e invitar a la participación. En algunos casos, el visitante se convierte en cocreador activo. En este escenario también han surgido dudas, por ejemplo si los artistas no controlan el resultado final de su obra, ¿sigue siendo arte?, ¿hay una pérdida de lo artístico cuando hay colaboraciones en la obra por parte de otros?.

\subsection{Diseño}

Si las exposiciones en museos tienen que sobrevivir a los grandes e irreversibles cambios sociales que conllevan los avances tecnológicos y la globalización además de los cambios de consumo, necesitaremos desarrollar o cambiar radicalmente nuestras prácticas expositivas en los próximos años. Preparados para la participación, los museos no deben adoptar simplemente las prácticas asociadas a los lugares de reunión social basadas en lo lúdico, ya que perderían su función y su identidad.

A raíz del desarrollo de la cultura participativa han surgido nuevas formas de diseño, una apuesta es el Diseño Centrado en las Personas (DCP) que comienza examinando las necesidades, los sueños y los comportamientos del público que se verá beneficiado por las soluciones resultantes ${ }^{21}$. Se valora hoy en día la innovación en la forma de presentación, la utilización de distintos medios y su integración para proporcionar nuevas experiencias que provocan respuestas.

\section{Conclusión}

Implantar técnicas de participación requiere de algunos cambios en los planteamientos institucionales sobre su autoridad y su relación con el público; éstos dependen del compromiso de la institución. Los profesionales de los museos no son simples transmisores del mundo, son activos participantes de cómo el mundo se percibe y se comprende, son participantes en la creación de significados y los que dan forma a la realidad. Los museos deben invitar a la colaboración para co-crear exposiciones, proporcionar maneras nuevas de mirar a los estereotipos y ofrecer lugares para detenerse. El papel de los profesionales de los museos es diseñar para crear múltiples experiencias, asegurarse que un gran número de personas puede conectar con los objetos e historias presentados. 


\section{Notas}

1. El lema del Día Internacional de los Museos de 2014 es Los vínculos creados por las colecciones de los museos. "En su papel de relatores del patrimonio cultural y la memoria colectiva de una comunidad, los museos contribuyen de manera importante al desarrollo de la sociedad, vinculando a los visitantes a los diferentes tipos de colecciones. El cual pretende renovar los métodos tradicionales de participación que han implementado los museos para mantener vivo el interés de las personas interesadas". Así se explica en el Comunicado de prensa del 15 de octubre de 2013.

2. En realidad se propone hacer balance del estado de las investigaciones en este campo, confrontar los análisis y los avances e interrogarse sobre las nuevas tendencias que se producen en el seno del campo museal. [URL: http://network.icom.museum/icofom/conferencias/proxima-conferencia/L/1/]. Acceso el 10/03/2014.

En el programa marco de investigación e innovación de la Unión Europea, Horizonte 2020, el Reto 6 apunta también en esta dirección, ya que se desarrolla bajo el lema "Europa en un mundo cambiante: sociedades inclusivas, innovadoras y reflexivas".

En otro contexto, la American Alliance of Museums definió varias tendencias significativas en el 2013 para los museos y sus comunidades que tienen que ver con el desarrollo y uso de la tecnología, -el uso positivo y el abuso- que ha favorecido por otra parte la apuesta por la desconexión y la contemplación, el cambio de los patrones de población y visitas a los museos, así como la financiación.

3. Definición de impacto según El Diccionario de la lengua española (DRAE), 2001: "Efecto producido en la opinión pública por un acontecimiento, una disposición de la autoridad, una noticia, una catástrofe, etc".

4. Stephen Weil ya lo expuso en 2002 cuando escribió "los profesionales de los museos necesitan recordar más enérgicamente de lo que generalmente hacen que los museos pueden mejorar sustancialmente las vidas de los individuos, incluso cambiarlas, y hacer de las comunidades mejores lugares para vivir".

5. Los museos siempre han ayudado a la población en tiempos difíciles, por ejemplo, cuando la Galería Nacional de Londres se mantuvo abierta durante la Segunda Guerra Mundial, o los museos de Estados Unidos que abrieron sus puertas de forma gratuita después del atentado del 11 de septiembre de 2001 y organizaron actividades para dar apoyo a la población. Ver Marjorie Schwarzer, 2006: 26.

6. La frase es del historiador Edward T. Linenthal, recuerda el título de Duncan F. Cameron aparecido en 1971 con el título "The Museum: a Temple or the Forum".

7. The Economist. "Museos. Templos del placer". 21 de diciembre de 2013. [URL: http://www.economist. $\mathrm{com} /$ news/special-report/21591707-museums-world-over-are-doing-amazingly-well-says-fiammetta-rocco-can-they-keep]. Acceso el 2/02/2014.

8. También planteaba que la equiparación de museo con casa de tesoros es una metáfora llevada al extremo que quiere decir que allí se guarda un patrimonio frágil y amenazado (1995: 9).

9. Op cit.

10. El Museo recibió a 3.184.640 visitantes en 2013, un 25\% más que en 2012. La exposición de Dalí celebrada del 27 abril al 2 septiembre de 2013 fue visitada por 732.339 personas. Otras cifras de visitantes se pueden consultar en [ URL: http:/www.arteinformado.com/Noticias/3855/fuertes-subidas-y-bajadas-en-las-cifras-de-visitantes-de-museos-en-2013-/?utm_source=boletin1\&utm_medium=email\&utm_ campaign=noticias]. Acceso el 1/03/2014.

Hay algunos informes, entre ellos el del National Endowment for the Arts (2009) sobre las visitas a instituciones artísticas en Estados Unidos que revelan datos interesantes sobre los visitantes a museos y galerías.

11. En el artículo del El Econimista.es "Exposiciones "mainstream", el imán del gran público" 06/01/2014 destacaba las declaraciones de Manuel Borja Villel, director del Reina Sofía, en las que decía que el problema general de la caída de público tiene que ver con la crisis económica. "Las programaciones son en general más modestas, hay menos medios, se reducen los presupuestos y, con ellos, la parte destinada a comunicación. Además, en Madrid está la bajada del turismo". [URL: http://ecodiario.eleconomista.es/interstitial/ volver/jh_en14/cultura/noticias/5435105/01/14/exposiciones-mainstream-el-iman-del-gran-publico.html]. Acceso el 1/03/2014.

12. La elección de esta generación para el estudio tiene que ver con la evidencia de que estos individuos son los que en la actualidad están transformando las comunidades, experimentando y persiguiendo el cambio social por medios que parecen oponerse a lo tradicional, pero que en realidad tienen que ver con la nueva manera 
de trabajar en colaboración, con transparencia, de forma interactiva y emprendiendo nuevos caminos, lo que afecta positivamente a las comunidades locales y también a nivel mundial. Es una generación que se ha formado con otros métodos de aprendizaje. Ver 2013 Millenial Impact Report patrocinado por The Case Foundation.

13. Según el informe de la Museum Association, los museos mejoran el bienestar de los individuos, aumentan la calidad de la vida de la mayoría de la gente mejorando su salud física y mental. Y como ya ha sido mencionado, en contrapartida, la participación activa del público mejora a los museos. También el informe Growing Young Minds: How Museums and Libraries Create Lifelong Learners de 2013 argumenta que los museos juegan un papel fundamental en el aprendizaje temprano; asimismo, las personas mayores que conforman el perfil de los voluntarios de los museos al constatar su influencia en la mejora de su comunidad experimentan una satisfacción personal que tiene influencia beneficiosa en su salud. Por último, en el artículo "Art Makes You Smart" publicado en el New York Times el 23 de noviembre de 2013 se planteaba que "visitar un museo de arte expone a los estudiantes a diversidad de ideas que les enfrenta a diferentes perspectivas de la condición humana. Ampliar el acceso al arte, bien a través de los programas en escuelas o a través de visitas a museos o galerías locales debe ser una parte fundamental del plan de estudios de cualquier escuela". [URL: http:// www.nytimes.com/2013/11/24/opinion/sunday/art-makes-you-smart.html?_r=0]. Acceso el 1/03/2014.

14. Doce años más tarde, Dubin especificará que son las exposiciones las que "solidifican" la cultura, la ciencia, la historia, la identidad y las visiones globalizadoras (2011: 479).

15. Weil decía que el museo es un medio neutro que puede ser usado por cualquiera para cualquier cosa (1995: $\mathrm{xv})$.

16. No se habla de interactividad, que es una característica de la tecnología, sino de participación que es una característica de la cultura.

17. En 2011 tuvo lugar el seminario Libraries and Museums in the Era of Participatory Culture en Salzburgo, reconociendo que es un tiempo crucial para los museos que necesitan reorientación y reinvención, se dieron una serie de recomendaciones para asegurar el máximo acceso e implicación en el trabajo de museos y bibliotecas en todo el mundo.

Nina Simon (2008) define institución cultural participativa como un lugar donde los visitantes pueden crear, compartir y conectar entre ellos gracias al contenido. La gran diferencia entre las técnicas del diseño tradicional y el participativo es que la información fluye entre instituciones y usuarios.

18. The Participatory Museum (2008) es una guía práctica para trabajar con los miembros de una comunidad y con los visitantes, con el objetivo de hacer de las instituciones culturales lugares más dinámicos, significativos y esenciales.

19. La figura del director de audience engagement comenzó a surgir comienzos del siglo XXI, y gran parte de su labor se apoya en la utilización de la Web (crowdfunding, crowdsourcing) para conseguir llevar a cabo los proyectos y de tecnologías digitales. Por ejemplo, las redes personalizadas y proactivas que pueden dar a la interacción un nuevo significado. Existe ya una revolución de todo lo que tiene que ver con las colecciones, su documentación, acceso y cuidado de las mismas. También las nuevas tecnologías pueden ayudar a conseguir datos que tienen que ver con el comportamiento del visitante y sus preferencias, lo que ayudaría a la toma de decisiones.

Una precisión que debemos hacer es sobre la pluralidad de los visitantes. Cada uno con su propio bagaje cultural, intelectual y estético, intereses, conocimientos, y expectativas hace imposible la simple referencia a la palabra público. Según Mieke Bal (2011: 525), hablar de públicos ayuda a evitar asociaciones generales y estimula el desarrollo de estrategias que facilitan interacciones entre los visitantes y los objetos expuestos.

20. En varios números del International Journal of the Inclusive Museum se pueden encontrar ejemplos de actividades en el museo que fomentaron la participación de la comunidad. Esta publicación aborda la cuestión clave del papel del museo como producto de los cambios sociales y plantea cómo el museo puede ser más inclusivo. Fundado dentro de la Inclusive Museum Knowledge Community, se empezó a publicar en 2008 en la Universidad de Illinois.

21. El Diseño Centrado en las Personas es un proceso y un conjunto de técnicas que se usan para crear soluciones nuevas para el mundo. Estas soluciones incluyen productos, servicios, espacios, organizaciones y modos de interacción. Ver Human-Centered Design Toolkit [URL: http://www.ideo.com/work/human-centered-design-toolkit]. Acceso el 4/03/2014. 


\section{REFERENCIAS Bibliográficas}

Achieve (2013): Millenial Impact Report. Achieve y The Chase Foundation, Indianapolis. [URL: http:// www.themillennialimpact.com]. Acceso el 13/03/2014.

American Alliance of Museums (2013): Trendswatch 2013. Back to the Future. American Alliance of Museums, Washington D.C.

BAL, M. (2011): Exposing the public. A Comparison to Museum Studies (S. Macdonald, ed.), Wiley-Blackwell, Malden.

Bennet, T. (1995): The Birth of the Museum. History, theory, politics. Routledge, London and New York (ed. 2006).

CAmeron, D. F. (1971): The Museum: a Temple or the Forum. Curator, The Museum Journal 14 (1): 11-24.

Dillenburg, E. (2011): What, if Anything, Is a Museum? Exhibitionist, spring '11: 8-13.

Dubin, S. C. (1999): Displays of Power. New York University Press, New York and London.

Dubin, S. C. (2011): Incivilities in Civil-(ized) Places: "Culture Wars" in Comparative Perspective. A Comparison to Museum Studies (S. Macdonald, ed.), Wiley-Blackwell, Malden: 477-493.

HernÁNDEZ Hernández, F. (2011): Museos, multiculturalidad e inclusión social. El Pensamiento Museológico Contemporáneo. II Seminario de Investigación en Museología. Buenos Aires, Consejo Internacional de Museos- ICOM: 407-417.

IDEo (2008): Human-Centered Design Toolkit. IDEO San Francisco. (Versión en español Diseño centrado en las personas. Kit de herramientas).

Institute of Museum and Library Services (2013): Growing Young Minds: How Museums and Libraries Create Lifelong Learners. IMLS, Washington, D.C.

Jenkins, H. (2009): Confronting the challenges of participatory culture: Media education for the $21^{\text {st }}$ century. MacArthur Foundation Report on Digital Media and Learning. The MIT Press, Cambridge, Mass.

Linenthal, E. T.; Engelhardt, T. (eds.) (1996): History Wars: The Enola Gay and Other Battles for the American Past. Metropolitan Books, New York.

Museums Association (2013): Museum Change Lives. The MA'S Vision for the impact of Museums. Museums Association, London.

National Endowment FOR THE ARTs (2009): 2008 survey of public participation in the Arts. NEA Research Report, 49 November 2009, Washington, D.C.

Roco, F. (2013): Come the Culture Vultures. The Economist 19.12.2013.

Samis, P.; Michaelson, M. (2013): Meaning-Making in Nine Acts. The Exhibitionist, Spring, 13: 54-59.

Schawarzer, M. (2006): 100 Years of Museums in America. American Association of Museums, Washington, D.C.

Simon, N. (2010): Participatory Museum. Museum 2.0. Santa Cruz.

WeIL, S. (1995): A cabinet of curiosities. Inquiries into museums and their prospects. Washington D.C.: Smithsonian Institution Press, Washington D.C.

WeIL, S. (2002): Making Museum Matter. Smithsonian Institution Press, Washington D.C. 